\title{
Ceiling of care decisions at an older person's mental health unit in Gloucestershire
}

\author{
Philip Slack, Anneka Rose \\ 2gether NHS Foundation Trust, UK
}

\begin{abstract}
This quality improvement project was inspired as an answer to a problem that many fellow psychiatric trainees had been struggling with while on-call covering the old age mental health hospital which includes a specialist dementia ward. The issue was that decisions around ceilings of care for patients were often not discussed or at least recorded in the electronic notes and as a result when reviewing deteriorating patients out of hours trainees would find themselves without any guidance on the treating medics opinion on what was in the best interests of the patient. This led to situations where unnecessary transfers to the acute hospital would occur overnight which could have been avoided with more consistent planning.
\end{abstract}

Prior to initiating the changes it was recorded that nine out of 47 inpatients had documented decisions on ceiling of care of treatment in the consultant's ward round entries. Next policies from acute hospitals were reviewed, opinions were discussed in departmental meetings, and eventually there was agreed a change in procedure with the consultant on the dementia ward around resuscitation and ceiling of care status and consistent recording of this.

Following the intervention there was seen an improvement in the recording of decisions around treatment and transfer of patients on the dementia ward of $80 \%$ (4/5) fully compliant with new criteria and then $71 \%(5 / 7)$ in successive cycles. Further communication both with relevant professionals on the old age ward and with the trainees on the on-call rota will be necessary to sustain any change but the centralised recording of resuscitation status and ceiling of care in the ward round entries have provided much more guidance than was previously available. In the future it may be possible to spread this policy throughout the entire old age mental health unit.

\section{Problem}

This project took place at an older persons mental health services (OPMHS) unit, 2gether NHS Foundation Trust, Gloucestershire, UK.

The feeling is that the root concern of the trainees on-call at the old age psychiatry unit relates to ceiling of treatment decisions. Trainees are often called to see someone with advanced dementia, very frail, with numerous medical co-morbidities who drops blood pressure/oxygen saturations in the middle of the night. There is very rarely a plan in place on the electronic notes system ("rio") from the day team as to whether or not transfer is in the patient's best interests. When speaking to medical registrar's etc, they frequently ask about ceiling of care which the on call doctor feels should not be their role. It seems unreasonable to expect an on call junior to be calling the next of kin out of hours to have these difficult conversations, when they could have been pre-empted by speaking to family during working hours by the team in regular contact with the patient and their family.

It was decided to plan a QI project to look at all the OPMHS admisisons as a snapshot and to work out what percentage of these patients have plans for event of deterioration in place. The main local acute hospitals trust has policy that by post take ward round a level of care decision should have been made and the OPMHS ward could implement a similar policy.
If such a system was in place with a standard that these discussions had occurred by the first ward round, ideally with family and the patient and a level recorded in progress notes in a consistent location, then it is strongly felt that none of the trainees would be raising concerns about having to make these difficult decisions out of hours. A lot of concern stems from lack of confidence in making these decisions and the pressure to decide overnight/weekends.

\section{Background}

As mentioned, the local acute trust has policy that by post take ward round a level of care decision should have been made. An example of this policy is below[1]:

1. Attempt CPR and refer to DCC if patient deteriorates

2. DNACPR but refer to DCC if patient deteriorates

3. DNACPR and do not refer to DCC if patient deterioratesgive active ward care

4. DNACPR and do not refer to DCC if patient deterioratespatient likely to be dying, consider palliative care referral.

A similar policy could be implemented, for example:

1. Attempt CPR, for full active treatment including transfer to general hospital

2. DNACPR but for full active ward based care including 
BMJ Quality Improvement Reports

transfer to general hospital

3. DNACPR, care to be given at OPMHS unit only

4. DNACPR, do not transfer to general hospital and consider palliative referral.

This QI project was the suggested to the director of medical education who approved this area of study. It was then discussed at the quarterly junior doctors' forum and received the backing of the medical director for the Trust as well as those trainees and consultants present.

\section{Baseline measurement}

The baseline measurements that were initially decided upon were to collect information from all of the inpatients on the three wards at the local OPMHS unit (two functional wards and one dementia unit) to give an overall idea of the situation. This captured results from 47 patients on $26 / 11 / 14$.

It was agreed to collect data on whether there was a ceiling of care decision made in the electronic notes system, both in the progress notes and on the alert system. The weekly MDT entries by the consultant were studied as well as searching all the notes with appropriate search terms, "ceiling" "advance" "transfer" "CPR" "DNAR" "DNR" "resus" "hospital", plus by searching for the hospitals by name. Not only was it considered whether this information was recorded in the notes, but also where it was recorded. The date the decision was made was also noted, along with whether it was flagged up on the front page of $\mathrm{RiO}$ in the alert section.

While searching for the above, there was also collected similar information on whether there was a recording of the patient's resuscitation status, as it was felt this was a connected decision with plans around whether patients are suitable for transfer to the acute trust. Admission dates were collated as well as dates that decisions were made at so it would be possible to assess the timeliness of these decisions. Also attached is the table of anonymous results below.

Initial analysis of the data showed:

Nine of 47 patients had a decision on ceiling of care documented somewhere in the electronic notes. Of these nine, there was little consistency where it was recorded and by whom. For example, it was mostly recorded in the progress notes although sometimes it was recorded in the ward round entry, and in addition sometimes as an alert on the system (seven of the nine). The status was usually recorded by the consultant but on a couple of entries the decision was documented by the junior doctor. The decision was not always particularly generalised and was often in relation to a particular physical illness at the time, therefore it wasn't clear whether it was still applicable.

An interesting contrast was the reporting of CPR status. Forty of 47 patients at the time of initial data collection had a clear CPR status documented. Two patients had contradictory entries regarding resuscitation status and therefore we contacted the relevant consultants so that this safety issue could immediately be rectified.

See supplementary file: ds4636.xIsx - "QIP baseline data"

\section{Design}

Following analysis of our initial data collection, there was a meeting to decide upon the best way to carry potential change forward. As a starting point it was felt that the dementia ward was the most important ward for ensuring that decisions around ceiling of care and CPR status to be consistently recorded and therefore it was decided to trial the change there.

The initial feeling was that if the consultant at the first ward round could document a decision on ceiling of care (even if in the vast majority of cases they may feel full active treatment is the best starting point) it would provide very important support to out of hours doctors trying to discuss patients that they are unfamiliar with with acute medical doctors in a different hospital.

It was thought that potential avenues would be to record this either as an alert on the front page (as is done with allergies and usually CPR status) or in the weekly ward round entries (which would have the benefit of having the backing of the entire multidisciplinary team and would be a prompt to review weekly).

It was then decided to arrange a meeting with the psychogeriatrician for the dementia ward to discuss these ideas, including discuss the protocol that is used at the acute trust (as previously documented).

\section{Strategy}

PDSA cycle 1: This PDSA cycle commenced with discussion with the consultant on the dementia ward regarding the initial data. It was highlighted that there were concerns regarding inconsistent or lack of ceiling of care documentation. A plan was agreed to document such decisions in MDT entries and if no data recorded the default is that the patient is for CPR and for active treatment including transfers. A month after this conversation new data was collected and analysed.

PDSA cycle 2: This cycle was completed two months after the initial discussion took place. There was continuation with plans to encourage spread of information via forums, meetings and emails. In cycle 2 it was hoped that there would be found at least a sustained level of recording, if not an improvement. Data was collected and analysed for all inpatients on the dementia ward.

See supplementary file: ds4695.docx - "PDSA Cycles 1 and 2"

\section{Post-measurement}

PDSA cycle 1: The records were analysed of all 14 patients on the dementia ward. Of the 14 in-patients on the ward, five were either new patients or had changes in their ceiling of care or resuscitation status compared with the baseline study. Therefore the other nine 
patients would show the same results as previously recorded. It was decided to consolidate the results into one category rather than separately recording DNAR status and ceiling of care. Then it was decided to use the categories previously suggested in this QIP, based on those used in the acute hospital. $0=$ full active treatment and CPR, $1=$ DNAR, full active treatment, $2=$ not for transfer, treatment on ward, $3=$ not for transfer, palliate.

It was also recorded when the latest change in this status was made by the MDT.

Of the five changes in status, the breakdown was as follows: two of the patients were for full active treatment, one of these was specified in the MDT notes (this was useful despite not being a requirement as would be the case by default) and the other had "for resuscitation" specified in the MDT notes.

One patient had been made "not for resuscitation" in the MDT notes and had also been specified in the MDT entry that the patient was still for transfer and active treatment.

Two patients were made "not for resuscitation" and "not for transfer to the general hospitals" but still "for ward level treatment". On one occasion this was recorded in the MDT notes, on the other unfortunately this was recorded as a separate note by the consultant and not in the MDT notes.

So of the five new statuses, four fully complied with our suggested changes and one partially complied (the conversation had occurred and was recorded but not in the ideal location. It was however easy to find in the notes). This was much better than first predicted.

PDSA cycle 2: 17 inpatients were on the dementia ward at the time of our second cycle. Of these patients, eight were either new patients or had changes in their resuscitation or ceiling of care status which had occurred after the first cycle of our QIP.

One of the eight patients had only been admitted a few hours previously and as such had not yet been reviewed by the consultant.

Two of the patients had been seen by the consultant and had been documented in the MDT notes that they were for CPR and therefore for full active treatment.

One of the patients who had previously been stated as for CPR for the past four months now had a MDT entry that specified that they were for full active treatment and transfer, though this is not necessary to specify according to the criteria previously agreed upon.

One of the patients had a consultant decision documented in the progress notes and alert system that they were not for CPR but were for transfer. Another patient had a progress note and alert note stating that they were not for CPR and not for transfer. Unfortunately these two were not recorded in the MDT notes as the consistent location agreed upon.
Following the previous cycle there was a change in one patient's ceiling of care level from not for CPR and not for transfer to not for CPR but for transfer. This was correctly recorded in the MDT notes.

Finally one new patient had been seen and a plan was agreed that they were not suitable for CPR or transfer to the acute hospital and this was correctly recorded in the latest MDT entry. It was also in the alert system and progress notes.

Excluding the newest patient (who had not yet been reviewed) five of the seven patients had their CPR status and ceiling of care suitably recorded in the MDT notes. Two patients did not have their status consistently recorded in the MDT entries but in the alerts and general notes. There was no change in the other nine patients on the ward, all of whom had been admitted prior to the first cycle of the QIP.

See supplementary file: ds4637.xlsx - "Copy of QIP PDSA cycle 1 and 2 anon"

\section{Lessons and limitations}

The take home message that was learnt from undertaking this project is that change can happen, but it is often slow. After doing the first data collection exercise to assess the situation prior to change, you could see that documentation was inconsistent and at times conflicting. As a result, this leads to the doctors reviewing patients out of hours becoming confused as to what the ceiling of care decision is and what would be in best interest of the patient.

It was initially tackled by speaking with the consultant on the dementia ward who agreed to document any decisions made in the weekly MDT section on RiO. If no decision was documented it can be assumed the team believe full treatment and active transfer to be in the patient's best interest.

It was hoped that there might be a possibility to introduce a similar policy to this in the acute trust where patients have ceiling of care documented by post take ward round, and that this could be the case by first MDT in the OPMHS unit. After speaking to the consultant he had felt that this would not be feasible as bringing in this policy would involve several meetings at senior levels in the trust and would also result in other policies being amended as a result. This is more of a long term goal as opposed to a quick change to improve service provision. Policy has just been changed in relation to capacity assessments and so to implement a policy on DNAR decisions at such time would be difficult.

It could be seen from the PDSA cycles that documentation had improved and that new patients being admitted were having clearer recording of decisions in both progress notes and in the MDT.

Turnover of patients is perhaps slower than anticipated at five to seven new patients per month on the ward, so each PDSA cycle often involved reviewing the same patients as they have remained on the ward several months. 


\section{BMJ Quality Improvement Reports}

\section{Conclusion}

The project was conducted initially due to concern from junior doctors on call about lack of clear documentation in the notes regarding ceiling of care decisions. It was first decided to look at available data and the conclusion was reached that documentation was indeed unclear in some cases, and it was discovered that conflicting decisions existed about resus status from progress notes and the MDT entries. This problem occurred since in the MDT proforma there is a section which states "DNAR status - for resus", so people were validating notes without actively changing this to "not for resus". This was highlighted to the teams very early on in the QIP prior to the first PDSA cycle, although unfortunately even by PDSA cycle 2 it was still found that this problem had occurred with two out of 17 patients on the dementia ward.

On a positive note, for the new patients being admitted you can see that documentation for the most part was clearer, with decisions on both resus and ceiling of care being recorded in MDT entries and on the alert system. This is a definite improvement that has occurred since undertaking this project and will result in much clearer documentation for both staff working in regular contact with the patient and for doctors reviewing the patient in an on call setting.

It was initially rather discouraging when it could be seen that errors in documentation were still occurring, however from completing two PDSA cycles it does seem that positive changes are taking place albeit slowly. The consultant on the dementia ward has since been contacted to recommend that on the MDT proforma that is used it says "DNAR status-" and is left blank as no documentation here would be a preference to wrong recording. Whoever is typing up the notes should take the responsibility of filling in the correct resus status when the meeting occurs.

The project has helped doctors on call to understand that if MDT notes do not comment on ceiling of care then the default is that the team feel transfer is in the patient's best interest and they are for active treatment. This message has been reiterated to the on call doctors at our quarterly junior doctors forum.

\section{References}

1. Gloucestershire Hospitals NHS Foundation Trust. Unwell/Potentially Deteriorating Patient Plan. September 2012 (Amended August 2013).

\section{Declaration of interests}

Nothing to declare.

\section{Acknowledgements}

Dr David Ogden, Dr Amjad Uppal. 